Poincare Journal of Analysis E Applications

Vol. 2015 (2), Special Issue (IWWFA-II, Delhi), 13-36

CPoincare Publishers

\title{
CONSTRUCTIONS OF MRA-BASED WAVELETS AND FRAMES IN WALSH ANALYSIS
}

\author{
Yu. A. Farkov \\ $\begin{array}{lll}\text { Date of Receiving } & : & 03.03 .2015 \\ \text { Date of Revision } & : & 14.05 .2015 \\ \text { Date of Acceptance } & : & 18.05 .2015\end{array}$
}

\begin{abstract}
In this paper, we review the basic constructions of MRA-based orthogonal wavelets and tight frames related to the generalized Walsh functions. Several differences between the constructed wavelets and the classical wavelets are indicated. Further, for any integer $p \geq 2$, all compactly supported step refinable functions on the Vilenkin group $G_{p}$ are characterized. In addition, we include references to the following topics: biorthogonal wavelets on $G_{p}$, approximation properties of compactly supported orthogonal wavelets and frames on the Vilenkin/Cantor groups, unconditional convergence of wavelet expansions, nonstationary, periodic, and discrete wavelets in Walsh analysis, $p$-multiresolution analysis on the positive half-line, and related subjects.
\end{abstract}

\section{Introduction}

Given an integer $p \geq 2$, the Vilenkin group $G_{p}$ consists of sequences $x=\left(x_{j}\right)$, where $x_{j} \in\{0,1, \ldots, p-1\}$ for $j \in \mathbb{Z}$ and with at most finite number of negative $j$ such that $x_{j} \neq 0$. The zero sequence is denoted by $\theta$. If $x \neq \theta$, then there exists a unique $k=k(x)$ such that $x_{k} \neq 0$ and $x_{j}=0$ for all $j<k$. The group operation $\oplus$ on $G$ is defined as the coordinatewise addition modulo $p$,

$$
\left(z_{j}\right)=\left(x_{j}\right) \oplus\left(y_{j}\right) \Longleftrightarrow z_{j}=x_{j}+y_{j}(\bmod p) \text { for all } j \in \mathbb{Z} ;
$$

the topology on $G_{p}$ is introduced via the complete system of neighbourhoods of zero

$$
U_{l}=\left\{\left(x_{j}\right) \in G_{p}: x_{j}=0 \text { for all } j \leq l\right\}, \quad l \in \mathbb{Z} .
$$

The equality $z=x \ominus y$ means that $z \oplus y=x$. For $p=2$ we have $x \oplus y=x \ominus y$ and the group $G_{2}$ coincides with the locally compact Cantor group $\mathcal{C}$.

Notice that for $p=2$ the subgroup $U_{0}$ is isomorphic to the compact Cantor group $\mathcal{C}_{0}$; i.e., the topological Cartesian product of a countable set of cyclic groups with discrete

2010 Mathematics Subject Classification. 42C40, 42C10, 42C15, 43A15, 65T60.

Key words and phrases. wavelets, frames, Walsh functions, Cantor group, Vilenkin groups, local fields.

The author thanks E. A. Rodionov for writing computer programs to draw graphs of wavelets and frames.

Communicated by. V. Yu. Protasov 